Thirdly, and perhaps most importantly, the telemedicine components that would be most crucial to improving adherence need to be better understood, so that the most efficient system can be designed. The current study used self-reported information and was focused on motivating patients to use CPAP. However, current technology can monitor CPAP pressures, leaks and objective compliance and send this information to the practitioner on a daily basis. Could early intervention using this information improve CPAP adherence even more? Alternatively, is it the regular automated telephone followups that are most important in motivating patients to use the device? Does the patient perception of being continuously monitored by their healthcare team (whether or not they actually are) serve as the main motivator for adherence?

Despite these caveats, we believe it likely that telemedicine will be integrated into the care of patients with OSA in the near future. The potential benefits in terms of monitoring, early intervention of problems, patient education, improving adherence and improving patient outcomes should outweigh technology costs. However, the most cost-efficient way to do this is yet to be determined.

Competing interests NA has received a research grant from Respironics Inc. MK has no competing interests.

Provenance and peer review Commissioned; not externally peer reviewed.

Published Online First 26 October 2010

Thorax 2010;65:1035-1036.

doi:10.1136/thx.2010.140897

\section{REFERENCES}

1. Al Lawati NM, Patel SR, Ayas NT. Epidemiology, risk factors, and consequences of obstructive sleep apnea and short sleep duration. Prog Cardiovasc Dis 2009;51:285-93.

2. George CF. Sleep. 5: driving and automobile crashes in patients with obstructive sleep apnoea/hypopnoea syndrome. Thorax 2004;59:804-7.

3. Marin JM, Carrizo SJ, Vicente E, et al. Long-term cardiovascular outcomes in men with obstructive sleep apnoea-hypopnoea with or without treatment with continuous positive airway pressure: an observational study. Lancet 2005:365:1046-53.
4. Engleman HM, Wild MR. Improving CPAP use by patients with the sleep apnoea/hypopnoea syndrome. Sleep Med Rev 2003;7:81-99.

5. Doherty LS, Kiely JL, Swan V, et al. Long-term effects of nasal continuous positive airway pressure therapy on cardiovascular outcomes in sleep apnea syndrome. Chest 2005;127:2076-84.

6. Zozula R, Rosen R. Compliance with continuous positive airway pressure therapy: assessing and improving treatment outcomes. Curr Opin Pulm Med 2001:7:391-8.

7. Reid J. A Telemedicine Primer: Understanding the Issues. Billings, MT: Innovative Medical Communications, 1996.

8. Taylor $\mathbf{Y}$, Eliasson A, Andrada T, et al. The role of telemedicine in CPAP compliance for patients with obstructive sleep apnea syndrome. Sleep Breath 2006; 10:132-8

9. Smith CE, Dauz ER, Clements F, et al. Telehealth services to improve nonadherence: a placebocontrolled study. Telemed J E Health 2006;12:289-96.

10. DeMolles DA, Sparrow D, Gottlieb DJ, et al. A pilot trial of a telecommunications system in sleep apnea management. Medical Care 2004:42:764-9.

11. Sparrow D, Aloia M, DeMolles DA, et al. A telemedicine intervention to improve adherence to continuous positive airway pressure: a randomised controlled trial. Thorax 2010;65:1061-6.

\title{
BCG vaccination: 90 years on and still so much to learn ...
}

\author{
Ajit Lalvani, Saranya Sridhar
}

The history of vaccination against tuberculosis abounds with instances of scientific discovery and rediscovery. At the end of the 19th and beginning of the 20th century, following Robert Koch's announcement of his discovery of $\mathrm{Myco}$ bacterium tuberculosis in 1882, scientists across the world, including Koch himself, set out to invent a vaccine against tuberculosis. In 1908 Leon Calmette, a bacteriologist, and Camille Guérin, a veterinarian at the Pasteur Institute, Lille began an experiment to devise a vaccine by attenuating a Mycobacterium bovis strain until it lost its virulence. Thirteen years and 230 passages later, they were able to show that

Tuberculosis Research Unit, Department of Respiratory Medicine, National Heart and Lung Institute, Imperial College London, London, UK

Correspondence to Ajit Lalvani, Tuberculosis Research Unit, Department of Respiratory Medicine, National Heart and Lung Institute, Imperial College London, Norfolk Place, London W2 1PG, UK;

a.lalvani@imperial.ac.uk the strain was protective in animal models and no longer caused disease which was subsequently discovered to be primarily due to the loss of the genes in the region of difference 1 (RD1) region of the $M$ bovis genome. ${ }^{1}$ That same year, an infant tuberculosis contact was given the first dose of this live attenuated vaccine, $M$ bovis Bacille Calmette-Geurin (BCG). Now, with over 3 billion doses administered, BCG is the most widely used vaccine worldwide.

Since its first use 90 years ago, BCG has been recommended as a vaccine because of its partial protective effect against active tuberculosis and death, albeit with greater efficacy against disseminated and meningeal disease in children than pulmonary disease in adolescents and adults. ${ }^{2} 3$ This, taken together with autopsy studies suggesting BCG decreases the size of pulmonary tuberculous foci thereby limiting bacillary multiplication and spread $^{4}$ and animal models where BCG vaccination reduces bacillary burden after
$M$ tuberculosis challenge but does not protect against infection, led to the longstanding dogma that BCG protects against dissemination and disease but not against infection. It was only 5 years ago that the first report ${ }^{5}$ of the ability of BCG to protect against the acquisition of infection changed our thinking about how BCG acts. The finding that BCG can act at an early stage in the pathway from tuberculosis exposure to disease (figure 1) has since been corroborated in community-based contact investigations in adults and children in Hamburg ${ }^{6}$ and in two studies investigating school tuberculosis outbreaks in the UK, ${ }^{7}$ the latest of which is published in the current issue of Thorax (see page 1067). ${ }^{8}$ Investigating a pointsource tuberculosis outbreak at a nursery school, Eriksen and colleagues found that BCG-vaccinated children were significantly less likely to be infected, as judged by interferon-gamma release-assay (IGRA) results, than unvaccinated children. Vaccine effectiveness against infection was estimated at $66 \%$ but, given the small size of the study, the CIs are sufficiently wide to overlap with the $38 \%{ }^{7}$ and $24 \%^{5}$ reduction in RR observed in the previous larger studies. Collectively, these data strongly suggest that at least part of the protective effect of BCG is attributable to protection against infection, which has substantial 


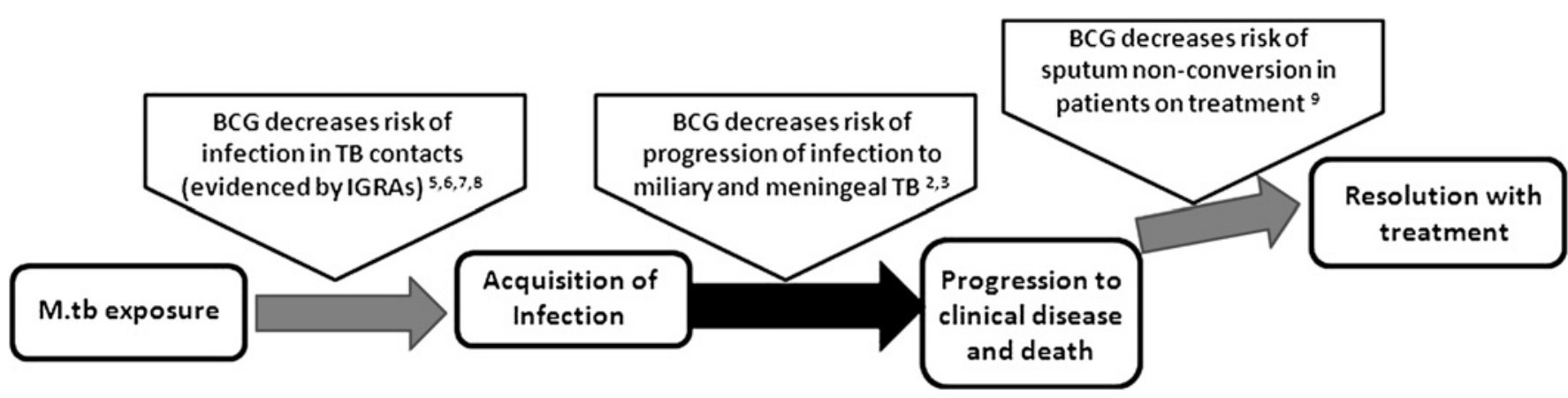

Figure 1 Schematic of the protective effects of BCG at different steps in the natural history of tuberculosis. Grey arrows represent effects of BCG discovered in the last 5 years while the black arrows represent effects historically known.

implications for the development and evaluation of new TB vaccines as well as the role of $\mathrm{BCG}$ in $\mathrm{TB}$ control programmes. Indeed Eriksen and colleagues discuss the case for a reconsideration of the threshold for BCG vaccination in regions with differing $T B$ incidence rates. However, conclusions about the causal role of BCG in protection against infection must be treated cautiously given the nonrandomised nature of the observational studies to date although, the cumulative evidence from the multiple epidemiologically distinct settings makes the case for the causal role of BCG increasingly compelling.

In this issue of Thorax we also learn about another hitherto unrecognised effect of BCG which appears to be its ability to aid treatment resolution in patients with active disease. Jeremiah and colleagues report results from a longitudinal study of 546 adult patients with generally high initial sputum bacillary loads undergoing treatment for pulmonary tuberculosis in Tanzania (see page 1072) ${ }^{9} ; 5.5 \%$ of sputum culture results failed to convert to negative after 2 months of treatment, a well-established marker of risk of relapse after treatment completion. On multivariate analysis, the absence of a BCG scar was associated with a highly significant threefold increased risk of sputum culture conversion failure. Patients with the highest initial sputum bacillary loads had a fivefold increased risk of 2 month sputum conversion failure as expected, while HIV status, CD4 count and body mass index were not significantly associated with sputum conversion failure. These results hint at a possible role for BCG in aiding the clearance of a large bacillary load during treatment. Considering that BCG does not protect against development of pulmonary tuberculosis in high-burden settings, its association with the microbiological response to treatment is unexpected and surprising. However, it is possible that antituberculous antibiotics and the host immune response cooperate in some way to reduce the mycobacterial burden in vivo, but the contribution of the host immune response per se is difficult to ascertain alongside the potent effect of antituberculous treatment in drug-sensitive disease. However, the significant rates of spontaneous recovery by patients with tuberculosis in the pre-antibiotic era in sanatoria ${ }^{10}$ and recent animal model data ${ }^{11}$ suggests a significant role for the host response in microbiological cure. Thus, it is at least plausible that host immunity plays a role in sputum bacillary clearance, but how this immunity is positively and durably modulated by BCG vaccination many years earlier is unknown. It is moreover perplexing that the presumably immunologically-mediated effects of BCG on the microbiological response to treatment could be so strong when HIV status and CD4 counts did not significantly influence sputum conversion rate. The paper by Jeremiah et al lacks information about the antibiotic susceptibilities of the infecting strains which are a major determinant of time to sputum smear and culture conversion but, given the reported low prevalence of multidrug-resistant tuberculosis in Tanzania, ${ }^{12}$ this is unlikely to have confounded the findings. This remarkable first report of the association of BCG vaccination with treatment resolution must be treated with caution until further studies corroborate this observation; nonetheless, the findings should encourage further research in a similar vein extended to children, extrapulmonary disease and drug-resistant organisms. Interestingly, this report has extended the evidence on the protective effects of BCG by identifying another much later point in the natural history of tuberculosis infection where BCG appears to act (figure 1).

As well as the increasing evidence that BCG affects several distinct points in the natural history of tuberculosis, mounting data attributes beneficial effects of BCG on diseases other than tuberculosis including leprosy, ${ }^{13}$ asthma, ${ }^{14}$ childhood pneumonia $^{15}$ and all-cause infant mortality. ${ }^{16} 17$ Thus, while we have been using BCG for almost a century, it is only more recently that an epidemiological evidence base is progressively revealing the pleiotropic effects of BCG on tuberculosis and other major diseases. Despite this recent progress in recognising the multiple effects of BCG, its immunological mechanisms of action as well as the reasons for its failure consistently to prevent adult pulmonary tuberculosis remain unclear. ${ }^{18}$ This is a major drawback, given the crucial importance of such understanding for the design and evaluation of new and improved tuberculosis vaccines with greater protective efficacy. Moreover, there are no less than twelve experimental vaccine candidates in clinical trials, most of which are being evaluated for their ability to boost immune responses originally primed by BCG. The problem is that we still do not know which of the immune responses induced by BCG mediate or correlate with protection against tuberculosis infection or disease. Although polyfunctional CD4 and CD8 $\mathrm{T}$ cells specific for $M$ tuberculosis antigens are increasingly viewed as potential correlates or mediators of protective immunity in vaccine trials and are induced by the new vaccines, ${ }^{19}{ }^{20}$ BCG-specific polyfunctional T cells do not correlate with protection against tuberculosis. ${ }^{21}$

A significant difficulty in interpreting the findings of both the papers published in this issue of Thorax and, indeed, all observational studies investigating the protective effects and underlying mechanisms of BCG is the reliance on scar formation as a surrogate marker for BCG vaccination. The dependence on scar formation to infer vaccine take is complicated by the high prevalence $(20-50 \%)$ of vaccine recipients unable to form 
a scar ${ }^{22-24}$ and the impact of vaccination technique on scar development. ${ }^{16}$ Moreover, scar formation does not correlate with BCG-induced development of adaptive cellular immune responses. ${ }^{25} 26$ This unresolved relationship between scar formation and BCG vaccination makes investigation of the true protective effect of the vaccine, independent of the scar, difficult to evaluate in an era where randomised trials of BCG versus placebo are unethical. The question is therefore whether the partial protection against the different stages in the path from tuberculosis exposure to disease conferred by BCG vaccination is mediated by immune responses that are independent of the response required for scarring or by immune responses that also cause the scar. A third possibility is that the scarring response to intradermal BCG is a surrogate marker of individuals who have preexisting inherent protective immunity to tuberculosis that is not induced by BCG vaccination. Longitudinal immunoepidemiological studies with clinical end points that follow BCG-vaccinated individuals who do and who do not scar are necessary to disentangle the immunological relationship between the triad of vaccination, scar formation and protection. Notably, large clinical end point vaccine trials designed to boost BCG with new vaccines may present an opportunity to resolve this thorny issue and identify true vaccine-induced correlates of protective immunity.

Like a fine wine that only gets better with age, 90 years after its first inoculation in humans we are still learning about the different facets of BCG in tuberculosis and beyond. Ironically, the scale of the global tuberculosis pandemic, itself partly a result of the inability of BCG fully to protect against tuberculosis, compels us to persist in attempting to unravel the mechanisms responsible for the partial effectiveness of BCG while simultaneously forging ahead with clinical trials of new experimental vaccines. Vaccination against tuberculosis would thus seem to have much in common with life itself, as surmised by Soren Kierkegaard: 'Life can only be understood backwards, but it must be lived forwards'.

Competing interests $\mathrm{AL}$ is inventor for several patents underpinning T cell-based diagnosis. The ESAT-6/CFP-10 IFN-gamma ELISpot was commercialised by an Oxford University spin-out company (T-SPOT.TB, Oxford Immunotec Ltd, Abingdon, UK) in which Oxford University and AL have minority shares of equity and entitlement to royalties. SS has no conflict of interest.

Provenance and peer review Commissioned; not externally peer reviewed.

Published Online First 21 October 2010

Thorax 2010;65:1036-1038.

doi:10.1136/thx.2010.140996

\section{REFERENCES}

1. Hsu T, Hingley-Wilson SM, Chen B, et al. The primary mechanism of attenuation of bacillus Calmette-Guerin is a loss of secreted lytic function required for invasion of lung interstitial tissue. Proc Natl Acad Sci U S A 2003;100:12420-5.

2. Trunz BB, Fine $P$, Dye $C$. Effect of $B C G$ vaccination on childhood tuberculous meningitis and miliary tuberculosis worldwide: a meta-analysis and assessment of cost-effectiveness. Lancet 2006;367:1173-80.

3. Colditz GA, Brewer TF, Berkey CS, et al. Efficacy of BCG vaccine in the prevention of tuberculosis. Metaanalysis of the published literature. JAMA 1994;271:698-702.

4. Sutherland I, Lindgren I. The protective effect of BCG vaccination as indicated by autopsy studies. Tubercle 1979;60:225-31.

5. Soysal A, Millington KA, Bakir M, et al. Effect of BCG vaccination on risk of Mycobacterium tuberculosis infection in children with household tuberculosis contact: a prospective communitybased study. Lancet 2005;366:1443-51.

6. Diel R, Loddenkemper R, Niemann $\mathrm{S}$, et al. Value of a whole-blood IGRA for developing active TB-an update. Am J Respir Crit Care Med. Published Online First: 27 Aug 2010. doi:10.1164/rccm.201006-09740C.

7. Eisenhut $\mathbf{M}$, Paranjothy S, Abubakar I, et al. BCG vaccination reduces risk of infection with Mycobacterium tuberculosis as detected by gamma interferon release assay. Vaccine 2009;27: 6116-20.

8. Eriksen J, Chow JY, Mellis V, et al. Protective effect of BCG vaccination in a nursery outbreak in 2009: time to reconsider the vaccination threshold? Thorax 2010;65:1067-71.

9. Jeremiah K, PrayGod G, Faurholt-Jepsen D, et al BCG vaccination status may predict sputum conversion in patients with pulmonary tuberculosis: a new consideration for an old vaccine? Thorax 2010;65:1072-6.

10. Millington KA, Gooding S, Hinks TS, et al. Mycobacterium tuberculosis-specific cellular immune profiles suggest bacillary persistence decades after spontaneous cure in untreated self-healed tuberculosis. J Infect Dis 2010;202:10.
11. Sada-Ovalle I, Skold M, Tian T, et al. Alpha-galactosylceramide as a therapeutic agent for pulmonary Mycobacterium tuberculosis infection. Am J Respir Crit Care Med 2010; 182:841-7.

12. Chonde TM, Basra D, Mfinanga SG, et al. National anti-tuberculosis drug resistance study in Tanzania. Int J Tuberc Lung Dis 2010;14:967-72.

13. Setia MS, Steinmaus C, Ho CS, et al. The role of BCG in prevention of leprosy: a meta-analysis. Lancet Infect Dis 2006;6:162-70.

14. El-Zein M, Parent ME, Benedetti A, et al. Does BCG vaccination protect against the development of childhood asthma? A systematic review and metaanalysis of epidemiological studies. Int J Epidemiol 2010;39:469-86.

15. Niobey FM, Duchiade MP, Vasconcelos AG, et al. [Risk factors for death caused by pneumonia in children younger than 1 year old in a metropolitan region of southeastern Brazil. A case- control study] Rev Saude Publica 1992;26:229-38.

16. Roth A, Gustafson P, Nhaga A, et al. BCG vaccination scar associated with better childhood survival in Guinea-Bissau. Int J Epidemiol 2005;34:540-7.

17. Garly ML, Martins CL, Bale C, et al. BCG scar and positive tuberculin reaction associated with reduced child mortality in West Africa. A non-specific beneficial effect of BCG? Vaccine 2003; 21:2782-90.

18. Orme IM. The Achilles heel of BCG. Tuberculosis (Edinb). Published Online First: 24 Jul 2010. doi:10.1016/j.tube.2010.06.002.

19. Abel B, Tameris M, Mansoor N, et al. The novel tuberculosis vaccine, AERAS-402, induces robust and polyfunctional CD4+ and CD8 + T cells in adults. Am J Respir Crit Care Med 2010;181:1407-17.

20. Beveridge NE, Price DA, Casazza JP, et al. Immunisation with BCG and recombinant MVA85A induces long-lasting, polyfunctional Mycobacterium tuberculosis-specific CD4+ memory T lymphocyte populations. Eur J Immunol 2007:37:3089-100.

21. Kagina BM, Abel B, Scriba TJ, et al. Specific T cell frequency and cytokine expression profile do not correlate with protection against tuberculosis, following BCG vaccination of newborns. Am J Respir Crit Care Med 2010;182:1073-9.

22. Fjallbrant H, Ridell M, Larsson LO. BCG scar and tuberculin reactivity in children and adults. Scand $J$ Infect Dis 2008;40:387-92.

23. Friedland IR. The booster effect with repeat tuberculin testing in children and its relationship to BCG vaccination. S Afr Med J 1990;77:387-9.

24. Faridi MM, Kaur S, Krishnamurthy $S$, et al. Tuberculin conversion and leukocyte migration inhibition test after BCG vaccination in newborn infants. Hum Vaccin 2009;5:690-5.

25. Jason J, Archibald LK, Nwanyanwu OC, et al. Clinical and immune impact of Mycobacterium bovis BCG vaccination scarring. Infect Immun 2002; 70:6188-95.

26. Rani SH, Vijayalakshmi V, Sunil K, et al. Cell mediated immunity in children with scar-failure following BCG vaccination. Indian Pediatr 1998;35:123-7. 